\title{
Preferences of Patients and Pharmacists with Regard to the Management of Drug-Drug Interactions: A Choice-Based Conjoint Analysis
}

\author{
Mette Heringa ${ }^{1,2,3}\left(\mathbb{D} \cdot\right.$ Annemieke Floor-Schreudering $^{1,2} \cdot$ Hans Wouters $^{4} \cdot$ \\ Peter A. G. M. De Smet ${ }^{5}$ Marcel L. Bouvy ${ }^{1,2}$
}

Published online: 30 September 2017

(c) The Author(s) 2017. This article is an open access publication

\begin{abstract}
Introduction The management of drug-drug interactions (DDIs) is a complex process in which risk-benefit assessments should be combined with the patient's perspective. Objective The aim of this study was to determine patients' and pharmacists' preferences regarding DDI management. Methods We conducted a choice-based conjoint survey about a fictitious DDI concerning the combination of a cardiovascular drug and an antibiotic for pneumonia. Patients and pharmacists had to choose 12 times between two management options. The options were described by five attributes, including risk, benefit and practical consequences. Each attribute could have two different levels, which were varied over the choice tasks. Latent class analysis was used to identify potential classes of respondents with distinct patterns of similar preferences.
\end{abstract}

Electronic supplementary material The online version of this article (doi:10.1007/s40264-017-0601-7) contains supplementary material, which is available to authorized users.

Mette Heringa

m.heringa@sirstevenshof.nl

1 SIR Institute for Pharmacy Practice and Policy, Theda Mansholtstraat 5B, 2331 JE Leiden, The Netherlands

2 Division of Pharmacoepidemiology and Clinical Pharmacology, Utrecht Institute for Pharmaceutical Sciences, Utrecht University, Utrecht, The Netherlands

3 Health Base Foundation, Houten, The Netherlands

4 Department of Pharmacotherapy, -Epidemiology and Economics, Groningen University, Groningen, The Netherlands

5 Departments of Clinical Pharmacy and IQ Healthcare, University Medical Centre St Radboud, Nijmegen, The Netherlands
Results In total, 298 patients and 178 pharmacists completed the questionnaire. The latent class model for both patients and pharmacists resulted in three classes. For patients, in one class the most importance was attached to avoiding switch of medication (class probability 20\%), in a second class to fewer adverse events (41\%), and in a third class to blood sampling (39\%). For pharmacists, again one class attached the highest importance to avoiding switch of medication (31\%). The other classes gave priority to curing pneumonia (31\%) and avoiding blood sampling (38\%). Conclusion The results showed diverging preferences regarding DDI management among both patients and pharmacists. Different groups attached different value to risk and benefit versus practical considerations. Awareness of existing variability in preferences among and between pharmacists and patients is a step towards shared decision making in DDI management.

\section{Key Points}

Risk-benefit assessments for drug-drug interaction management should incorporate the patient's perspective.

Both patients and pharmacists have diverging preferences regarding drug-drug interaction management.

The development and application of drug interaction management recommendations can benefit from the awareness of existing variability of preferences. 


\section{Introduction}

Clinical risk management of drug-drug interactions (DDIs) is complex. The management recommendations for DDIs often provide healthcare professionals with several options (e.g. additional monitoring, switch to an alternative drug, dose adjustment). These recommendations are generally based on the principle of evidence-based medicine and are dominated by risk-benefit assessments [1, 2]. In DDI management, these assessments are difficult because at least two drug therapies are involved and because the evidence for the different management options is generally limited. Moreover, recognizing the importance of shared decision making, the patient's perspective should be included [3-5]. In shared decision making, patients and healthcare professionals make healthcare decisions together, taking into account both scientific evidence and the patient's values and preferences [6].

The Grading of Recommendations Assessment, Development and Evaluation (GRADE) approach offers an evidence-to-decision framework for clinical decisions [7, 8]. The aspects involved in DDI decision making and clinical decision making in general are similar, with a complex risk-benefit balance of the therapeutic options being central in the assessment, and with uncertainty being high. Therefore, the GRADE approach has also been proposedand used-for the assessment of DDI management [1,9]. The GRADE model includes, in addition to aspects such as risk and benefit, variability in how patients value the main outcome. The acceptability and the feasibility of a recommendation for patients and healthcare providers are also part of the model. Thus, it is recognized that patients' values and preferences and their variability are relevant in the development of DDI management recommendations.

In addition to the role of patients' preferences in the development of recommendations, the patients' perspective should be taken into account in the application of recommendations in daily practice $[5,10]$. Currently, DDI management by pharmacists and physicians usually does not explicitly involve the patient's perspective. Little is known about patients' preferences in the field of DDI management and about potential incongruence with professionals' preferences. Based on investigations of patient preferences in other drug-related issues, variability among patients could be expected [11-13]. Both patients' and healthcare providers' perspectives may influence the choice of a specific DDI management option. Insight into these perspectives is useful for shared decision making in this field. The responsibility for DDI management is shared between the physician and the pharmacist but is a main focus for pharmacists. Therefore, we aimed to investigate patients' and pharmacists' preferences with regard to DDI management.

\section{Methods}

\subsection{Study Design}

A structured online questionnaire was administered to patients and community pharmacists. The core task was a choice-based conjoint (CBC) task on a fictitious DDI regarding a patient using cardiovascular medication who was in need of an antibiotic because of suspected pneumonia. In the $\mathrm{CBC}$, respondents had to choose between hypothetical options to determine the characteristics of an option to which they attached value (see Sect. 2.3). The CBC task was essentially the same for pharmacists and patients. Patients were asked to choose for themselves; pharmacists were asked to choose for a patient in general. The complete pharmacist questionnaire comprised sociodemographic characteristics, the CBC task, and an open question on situations or patient groups in which the preferences of the pharmacist would differ from the choices made in the CBC. The complete patient questionnaire comprised four parts: general questions on sociodemographic characteristics and drug use, the CBC task, a questionnaire on numeracy and a questionnaire on health literacy.

\subsection{Participants and Protocol}

Pharmacists were recruited from the Utrecht Pharmacy Practice Network for Education and Research (UPPER), which includes two-thirds of the 1900 Dutch community pharmacies [14]. The usual response rate in this network is $10-15 \%$. Email invitations included the URL of the online questionnaire, and a reminder was sent after 1-2 weeks.

Patients were recruited via a convenience sample of five community pharmacies from different regions in the Netherlands. Patients who were using cardiovascular drugs were selected to ensure that the fictitious DDI presented would be plausible. The patient selection was made from the pharmacy information system, which contains electronic patient records including a medication dispensing history and a coded registration of chronic conditions. A sample of 200 patients per pharmacy was randomly selected out of the patients who met the following inclusion criteria:

1. Aged $>40$ years

2. Use of cardiovascular medication based on dispensing data. We considered the following cardiovascular medication (anatomical therapeutic chemical [ATC] class [15]): lipid-modifying agents (C10), plateletaggregation inhibitors (B01AC) and antihypertensive drugs ( $\mathrm{C} 03$ diuretics, $\mathrm{C} 07$ beta-blocking agents, $\mathrm{C} 08$ calcium channel blockers or $\mathrm{C} 09$ renin angiotensin system inhibitors) 
3. Registered indication for cardiovascular risk management: hypertension, heart failure, coronary disease, diabetes mellitus or stroke

4. Available email address

5. No known terminal illness or impaired cognition

Patients were invited via email by their own pharmacist, with a reminder after 1-2 weeks.

\subsection{Choice-Based Conjoint Task}

Conjoint analyses and discrete-choice experiments, such as $\mathrm{CBC}$, are increasingly used in healthcare to elicit and quantify respondents' preferences [16, 17]. In CBC, respondents choose between hypothetical options that systematically vary in the value (level) of selected attributes, which reflects issues relevant to the decision. A CBC task was developed in accordance with guidelines [18-20], using Sawtooth Software (Lighthouse Studio version 9.2.0, Orem, UT, USA). The CBC was based on a fictive case illustrative of DDIs (Fig. 1), and plausible for a broad group of respondents: cardiovascular medications are among the most frequently used drugs and they often cause DDIs [21]. In the first step of the development of the CBC task, realistic attributes and levels were preselected (Table 1), representing four common DDI management options that could be applied after consultation between prescriber, pharmacist and patient: (1) no action, use both drugs concurrently; (2) replacement of the medicine the patient is already using; (3) replacement of the newly prescribed medicine; (4) additional monitoring such as blood testing [1]. The case, attributes and levels were selected based on considerations relevant in the development of drug interaction management guidelines [1, 8, 9] and the content of DDI management guidelines [22, 23]. This preselection was carried out by the research group, comprising four pharmacists experienced in pharmacy practice research and DDI assessment (one being a practising community pharmacist) and one psychologist. Second, the preselection of attributes was verified in five focus group meetings with a total of 38 patients using cardiovascular drugs, which were part of a parallel running investigation (manuscript in preparation). The focus groups were held in five community pharmacies and based on a DDI case very similar to the example in the CBC task. Patients were asked for their preferences in DDI management and the underlying rationale. Focus groups were audiotaped, transcribed, and analysed using inductive analysis of themes. The rationales expressed by the patients were assessed for potentially relevant attributes for the CBC task. Based on the focus groups, no new attributes were added, but a cost attribute was excluded. In the Netherlands, this attribute is not relevant for patients with chronic conditions because costs are covered by insurance, and therefore the cost component was experienced as unrealistic and confusing by the patients participating in the focus groups.

In the third step, the questionnaire was pre-tested by patients and pharmacists for understanding, feasibility and

Imagine: you suffer from pneumonia. Your general practitioner proposes to start a 10 day antibiotic course. In combination with your cardiovascular medicine, this can cause muscle pain. And in rare cases serious muscle problems with permanent damage. There are several options to manage this risk of muscle problems.

\section{Do you prefer option A of B?}

option A

\begin{tabular}{|c|c|c|}
\hline $\begin{array}{r}\text { switch to other cardiovascular drug } \\
\text { blood sampling twice during antibiotic course } \\
\text { serious muscle problems with permanent damage }\end{array}$ & $\begin{array}{l}\text { no } \\
\text { yes } \\
\text { in } 3 \text { out of } 10,000 \text { people }\end{array}$ & $\begin{array}{l}\text { yes } \\
\text { no } \\
\text { in } 1 \text { out of } 10,000 \text { people }\end{array}$ \\
\hline cure of pneumonia without hospitalization & in 90 out of 100 people & in 90 out of 100 people \\
\hline moderate muscle pain & in 5 out of 100 people & in 10 out of 100 people \\
\hline & 0 & 0 \\
\hline \multicolumn{2}{|c|}{$\begin{array}{l}\text { Level identical for both options } \\
\text { (indicated with different coloring) }\end{array}$} & Levels - \\
\hline
\end{tabular}

Fig. 1 Annotated example of choice set for patients. Example of a choice set, starting with a short description of the case (wording for patients). The choice set shows two options; respondents have to choose either option A or option B. For the complete questionnaire, see Electronic Supplementary Material 1 
Table 1 Attributes and levels

\begin{tabular}{|c|c|c|c|}
\hline Attribute & Explanation & Levels & $\begin{array}{l}\text { Corresponding DDI } \\
\text { management option }\end{array}$ \\
\hline \multirow[t]{2}{*}{$\begin{array}{l}\text { Serious muscle problems } \\
\text { with permanent damage }\end{array}$} & \multirow[t]{2}{*}{ The risk of serious muscle problems with permanent damage } & $\begin{array}{l}\text { In } 1 \text { of } \\
10,000 \\
\text { people }\end{array}$ & $\mathrm{MO} 2, \mathrm{MO} 3, \mathrm{MO} 4$ \\
\hline & & $\begin{array}{l}\text { In } 3 \text { of } \\
10,000 \\
\text { people }\end{array}$ & MO1 \\
\hline \multirow{2}{*}{$\begin{array}{l}\text { Blood sampling twice during } \\
\text { antibiotic course }\end{array}$} & \multirow{2}{*}{$\begin{array}{l}\text { Whether or not blood is sampled twice during the antibiotic course. } \\
\text { By blood testing, muscle problems can be diagnosed at an early } \\
\text { stage, before they become serious }\end{array}$} & No & $\mathrm{MO} 1, \mathrm{MO} 2, \mathrm{MO} 3$ \\
\hline & & Yes & MO4 \\
\hline \multirow[t]{2}{*}{$\begin{array}{l}\text { Curing pneumonia without } \\
\text { hospitalization }\end{array}$} & \multirow[t]{2}{*}{ The chance of recovering from pneumonia without hospitalization } & $\begin{array}{l}\text { In } 95 \text { of } 100 \\
\text { people }\end{array}$ & MO1, MO2, MO4 \\
\hline & & $\begin{array}{l}\text { In } 90 \text { of } 100 \\
\text { people }\end{array}$ & MO3 \\
\hline \multirow[t]{2}{*}{ Moderate muscle pain } & \multirow[t]{2}{*}{$\begin{array}{l}\text { The risk of moderate muscle pain. With moderate muscle pain, you } \\
\text { are limited in your daily activities (work, hobby) }\end{array}$} & $\begin{array}{l}\text { In } 5 \text { of } 100 \\
\text { people }\end{array}$ & MO2, MO3 \\
\hline & & $\begin{array}{l}\text { In } 10 \text { of } 100 \\
\text { people }\end{array}$ & MO1, MO4 \\
\hline \multirow{2}{*}{$\begin{array}{l}\text { Switch to other } \\
\text { cardiovascular drug }\end{array}$} & \multirow{2}{*}{$\begin{array}{l}\text { Whether or not you switch to another cardiovascular medicine, instead } \\
\text { of the one you are using at the moment }\end{array}$} & No & MO1, MO3, MO4 \\
\hline & & Yes & MO2 \\
\hline
\end{tabular}

$D D I$ drug-drug interaction, $M O$ management option

${ }^{a}$ MO1: no action, use both drugs concurrently; MO2: replacement of the medicine the patient is already using; MO3: replacement of the newly prescribed medicine of choice; or MO4: extra monitoring by blood testing

wording. A number of 12 choice sets was chosen to limit the time needed to complete the questionnaire to $10 \mathrm{~min}$ for pharmacists and to $15 \mathrm{~min}$ for patients (the patient questionnaire took longer because of the additional parts about health literacy and numeracy). The questionnaire was adapted according to the feedback in a cyclic process (two respondents per target group per cycle), until after three cycles no new issues were identified and good understanding was reached.

In the final questionnaire, the participating patients and pharmacists had to respond to 12 choice sets with two DDI management options each. Because of the complexity of the subject, the number of options was limited to two per choice set; no opt-out option was available, consistent with reality. The options to choose from were characterised by a full profile of five attributes. The attributes were presented in the same order for any given respondent, but the order was randomised between respondents. A balanced overlap design (with level balance and near orthogonality) was used to create the choice sets $[18,24]$. We generated 20 different combinations of 12 choice sets, which were randomly assigned to the respondents. The CBC was preceded by an explanation of the case and the choice task; a pop-up with additional information on the attributes and levels was available during the CBC. For a translated example of a choice set, see Fig. 1; for the complete CBC task, see Electronic Supplementary Material (ESM) 1.

\subsection{Health Literacy and Numeracy Measurement}

Measures of health literacy and numeracy were included to detect potential associations with preferences, as literacy and numeracy can influence decision making [25-29]. The Dutch versions of the validated Functional Communicative and Critical Health Literacy Scales (FCCHL) and the Subjective Numeracy Scale (SNS) were used.

The FCCHL comprises 14 statements about handling health information [30, 31]. The items are divided over three subscales: functional health literacy (understanding health information, five items), communicative health literacy (finding and using health information, five items) and critical health literacy (assessing health information, four items). Each item is rated on a 4-point scale, ranging from 1 (never/easy) to 4 (often/hard). After reversion of all scores, the scores on the items in a (sub)scale were summed and divided by the number of items in the scale to calculate a scale score (theoretical range $1-4$, with a higher score indicating higher health literacy).

The SNS comprises eight statements about respondents' performance and preferences with regard to handling numeric information in daily life. All items are rated on a 6-point scale, ranging from 1 (not at all good/never) to 6 (extremely good/very often) [27, 28, 32]. After reversion of item 7 , the total score was calculated by summing the item scores and dividing them by eight. The theoretical range is 
1-6, with a higher score indicating higher numeracy. For the complete FCCHL and SNS, see ESM 1.

\subsection{Sample Size}

Because of the exploratory nature of this study and the potential variability, an a priori estimation of effect size was not available. Taking into account general guidelines for CBC, the targeted sample size was 200 patient respondents and 200 pharmacist respondents $[18,19]$. The aim of our study was to obtain a general overview of preferences and potential variability in DDI management based on a fictitious case rather than an exact estimate of choice behaviour in DDI management.

\subsection{Data Analysis}

We used Microsoft ${ }^{\circledR}$ Excel 2010 and SPSS version 20.0 (SPSS Inc., Chicago, IL, USA) for descriptive statistics of basic characteristics. For non-responders analysis for patients, differences between groups were analysed using the $t$ test for normally distributed continuous variables and Pearson's Chi squared test for categorical variables. $P$ values $<0.05$ were considered statistically significant. Only completed questionnaires were included in further analysis.

The CBC data were analysed using Sawtooth Software. $\mathrm{CBC}$ analysis results in utilities, which are similar to a regression coefficient and represent the relative attractiveness (preference) of an attribute level and therefore its relative influence on the respondents' choice. Positive utilities reflect the preferred level; higher values reflect greater attractiveness. The importance of an attribute [relative importance (RI)], i.e. how much it contributes to the respondents' choice, is calculated by dividing the absolute value of the utility by the sum of the absolute value of all utilities.

We analysed the CBC data using latent class analysis [33] to examine the presence of classes (subgroups) of respondents with different preferences. In latent class analysis, the classes are 'latent': they are derived from distinct patterns of similar preferences in the data. Successive latent class models were estimated for one to five classes. The most likely number of classes was evaluated by assessing the goodness-of-fit indices for the model. Various goodness-of-fit statistics are available for latent class analysis, which we inspected in conjunction with each other. We assessed the LogLikelihood, McFadden's pseudo $\rho^{2}$ (value between 0.2 and 0.4 indicates good fit), Akaike information criterion (AIC; lower values indicate better fit) and Bayesian information criterion (BIC; lower values indicate better fit) $[20,33]$. In addition, the pattern of utilities for every model was assessed. Moreover, mean class probabilities (the mean of the probability that a respondent belonged to this class) were taken into account to maintain clinically relevant class sizes. Based on these considerations, the most likely number of classes (optimal model) was established for both patients and pharmacists. Subsequently, we tested whether every attribute significantly contributed to the model by a Chi squared test on the $-2 \operatorname{LogLikelihood~based~on~the~difference~between~the~}$ model with and without every single attribute.

For the final model, every respondent was assigned to the class for which she/he had the highest probability, and a comparison between classes was made with respect to the respondents' basic characteristics in SPSS. Analysis of variance (ANOVA) was used for normally distributed continuous variables and Pearson's Chi squared test for categorical variables. $P$-values $<0.05$ were considered statistically significant.

\section{Results}

\subsection{Respondents}

An invitation was sent to 1312 pharmacists between September and December 2016. The questionnaire was accessed by $236(18.0 \%)$ and completed by $178(13.5 \%)$ pharmacists. See Fig. 2 for a flowchart of this process and Table 2 for respondents' basic characteristics.

Five pharmacists selected 200 eligible patients each; invitations were emailed to 1000 patients between September and December 2016. The online questionnaire was accessed by 393 (39.3\%) and completed by 298 (29.8\%) patients. Between patients who completed the questionnaire $(n=298)$ and patients who filled out the basic characteristics but did not complete the questionnaire $(n=79)$, there were differences with respect to age (mean age 64.4 vs. 67.9 years; $p=0.01)$ and educational level [low $26.9 \%(n=79)$ vs. $48.0 \%(n=36)$; medium $39.1 \%$ $(n=115)$ vs. $33.3 \%(n=25)$; high $34.0 \%(n=100)$ vs. $18.7 \%(n=14) ; p<0.01]$. No differences were seen with respect to sex, duration of use of cardiovascular drugs and number of medicines in use.

\subsection{Latent Class Analysis}

The latent class analysis for both patients and pharmacists resulted in a three-class model. McFadden's pseudo $\rho^{2}$ was 0.24 for patients and 0.37 for pharmacists. Fit statistics indicated improvement in model fit with each additional class (see ESM 2) but with decreasing gain in model fit. With more than three classes, small classes and unclear differentiation arose compared with the number of respondents and attributes. The three-class models were 
Fig. 2 Flowchart respondents

\section{PATIENTS}

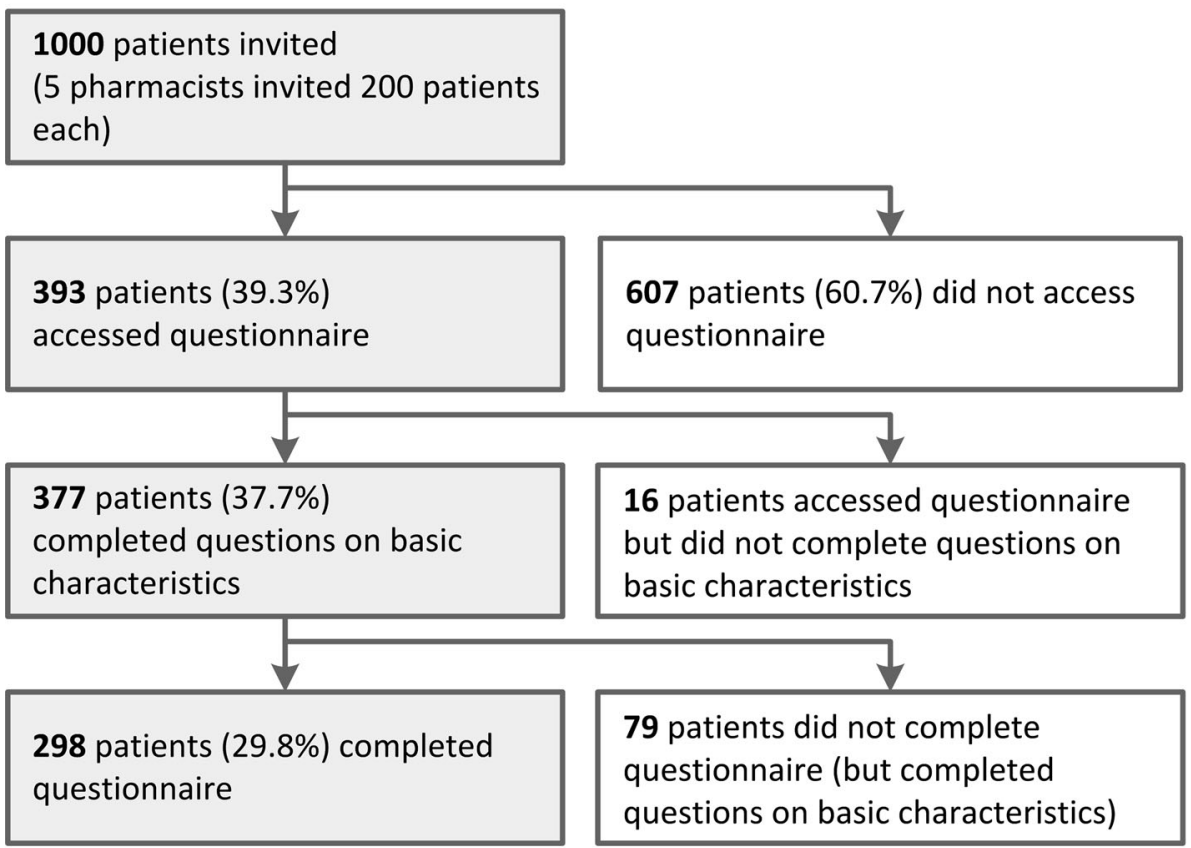

PHARMACISTS

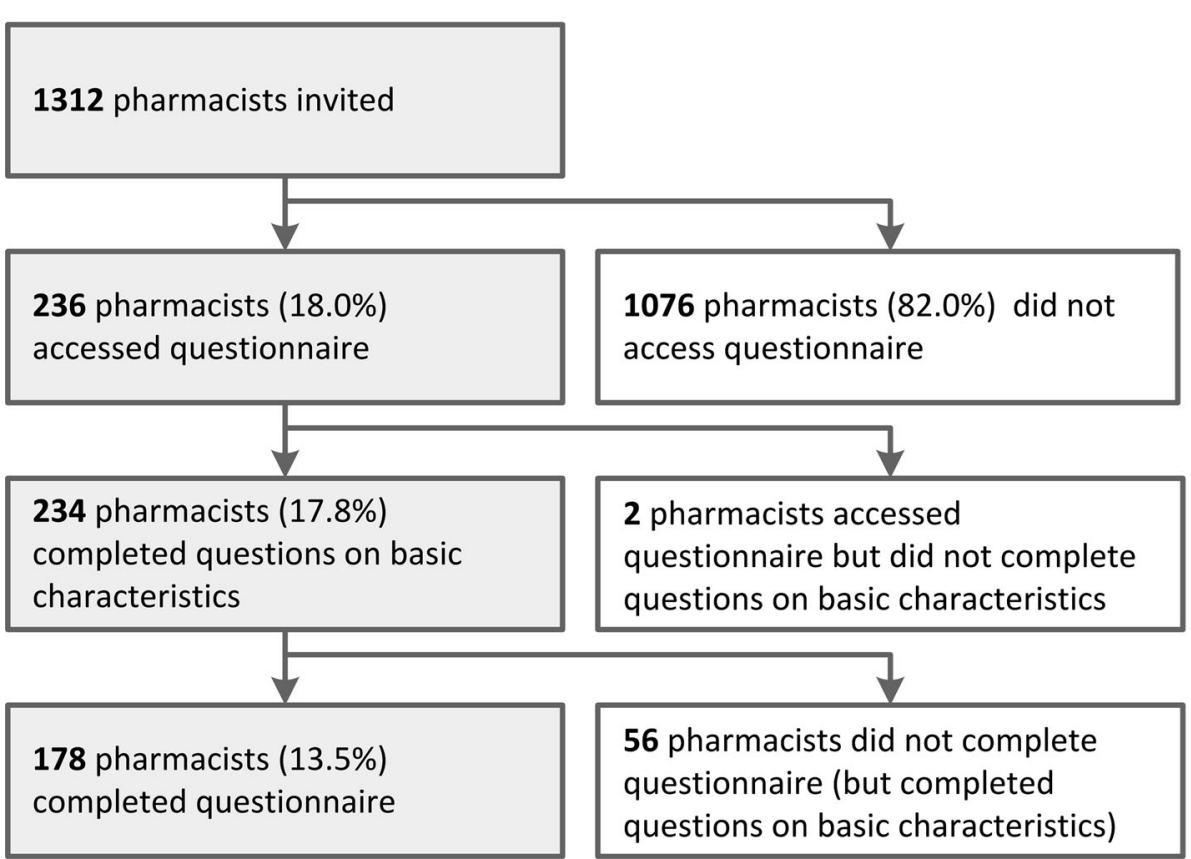

stable and had an average maximum class membership probability of $89.0 \%$ and $89.8 \%$ for patients and pharmacists, respectively. All attributes significantly contributed to the model for both pharmacists and patients.

For patients, level utility and attribute importance are shown per class in Table 3 . In all classes, a lower risk of muscle damage was preferred over a higher risk, a lower risk of moderate muscle pain was preferred over a higher risk, and a higher chance of curing pneumonia was preferred over a lower chance (reflected by positive utilities for the preferred level and negative utilities for the nonpreferred level). Preferences were less consistent for blood 
Table 2 Descriptive characteristics of respondents

\begin{tabular}{|c|c|c|}
\hline \multicolumn{2}{|l|}{ Patients } & \multirow{2}{*}{$\begin{array}{l}n=298 \\
64.4(9.6)\end{array}$} \\
\hline Age & Mean (SD) & \\
\hline Sex & Male & $186(62.4 \%)$ \\
\hline \multirow[t]{3}{*}{ Educational level $^{\mathrm{a}}$} & Low & $79(26.9 \%)$ \\
\hline & Medium & $115(39.1 \%)$ \\
\hline & High & $100(34.0 \%)$ \\
\hline Number of medicines in use & Mean (SD) & $4.7(2.7)$ \\
\hline \multirow[t]{2}{*}{ Duration of use of cardiovascular medicines } & $<=5$ years & $27.9 \%$ \\
\hline & $>5$ years & $72.1 \%$ \\
\hline SNS & Mean (SD) & $4.2(0.8)$ \\
\hline FCCHL total & Mean (SD) & $2.9(0.5)$ \\
\hline FCCHL functional & Mean (SD) & $3.0(0.5)$ \\
\hline FCCHL communicative & Mean (SD) & $3.2(0.6)$ \\
\hline FCCHL critical & Mean (SD) & $2.6(0.7)$ \\
\hline \multicolumn{2}{|l|}{ Pharmacists } & $n=178$ \\
\hline Age & Mean (SD) & $43.2(11.2)$ \\
\hline Sex & Male & $77(43.3 \%)$ \\
\hline \multirow[t]{3}{*}{ Years of practice in community pharmacy } & $0-5$ & $36(20.2 \%)$ \\
\hline & $6-15$ & $57(32.0 \%)$ \\
\hline & $>15$ & $85(47.8 \%)$ \\
\hline \multirow[t]{3}{*}{ Location of pharmacy } & Village (up to 20,000 inhabitants) & $71(39.9 \%)$ \\
\hline & Town $(20,000-150,000$ inhabitants $)$ & $61(34.3 \%)$ \\
\hline & City (over 150,000 inhabitants) & $46(25.8 \%)$ \\
\hline \multirow[t]{2}{*}{ Type of community pharmacy } & Community health centre & $92(51.7 \%)$ \\
\hline & Other & $86(48.3 \%)$ \\
\hline
\end{tabular}

FCCHL Functional Communicative and Critical Health Literacy Scales, SD standard deviation, SNS subjective numeracy scale

${ }^{a}$ Educational level was categorized as low (primary education or lower secondary), medium (intermediate/higher secondary or intermediate vocational), or high (higher vocational/university); data were missing for four respondents

sampling and switch of cardiovascular drug. In the first class, the most importance was attached to avoiding a switch of the cardiovascular drug in use (RI 58.9\%), followed by minimizing the risk of muscle damage (RI $21.9 \%$ ). This class was labelled 'stability focused'. In the second class, the most value was attached to minimizing the risk of muscle damage (RI 49.7\%) and maximizing the chance of curing pneumonia without hospitalization (RI 24.3\%). This second class was labelled 'risk focused'. In the third patient class, labelled 'certainty focused', options with blood sampling were preferred over options without (RI blood sampling 30.5\%). The patients' descriptive characteristics shown in Table 2 were not significantly associated with class assignment (see ESM 3a).

The pharmacists' results are shown in Table 4. In all classes, lower risks of harm were preferred over higher risks, higher chances of cure were preferred over lower chances, and avoidance of blood sampling and of switch of current medication was preferred. In the first class, the highest importance was attached to the risk of muscle damage (RI 40.6\%) and the chance of curing pneumonia without hospitalization (RI 41.4\%). This first pharmacist class was labelled 'risk focused'. In the second class, maintaining the current cardiovascular medication was valued most (RI 61.2\%); this class was labelled 'stability focused'. In the third class, priority was given to avoidance of blood sampling (RI 38.8\%), followed by avoidance of the switch of current cardiovascular medication (RI $25.2 \%$ ) and a low risk of muscle damage (RI 20.0\%). This pharmacist class was labelled 'practicality focused'. In a univariate analysis, pharmacist age, sex and years of practice were all associated with class assignment (respectively, $p=0.01, p=0.00 ; p=0.02$ ); see ESM $3 b$. The 'risk focused' pharmacists were the oldest, had been in practice for the most years, and were more often male. The 'stability focused' pharmacists were the youngest, had been in practice for the least years, and were more often female. 
Table 3 Results of patients' latent class analysis

\begin{tabular}{|c|c|c|c|c|c|c|c|}
\hline \multirow[t]{2}{*}{ Attribute } & \multirow[t]{2}{*}{ Level } & \multicolumn{2}{|c|}{$\begin{array}{l}\text { Class 1: 'stability focused' } \\
(20.3 \%)^{\mathrm{a}}\end{array}$} & \multicolumn{2}{|c|}{$\begin{array}{l}\text { Class 2: 'risk focused' } \\
(41.0 \%)^{\mathrm{a}}\end{array}$} & \multicolumn{2}{|c|}{$\begin{array}{l}\text { Class 3: 'certainty } \\
\text { focused' }(38.7 \%)^{\mathrm{a}}\end{array}$} \\
\hline & & Utility (SE) & RI & Utility (SE) & RI & Utility (SE) & RI \\
\hline \multirow[t]{2}{*}{ Muscle damage } & 1 of 10,000 & $0.62(0.09)^{\mathrm{b}}$ & $21.9 \%$ & $1.39(0.07)^{\mathrm{b}}$ & $49.7 \%$ & $0.21(0.04)^{\mathrm{b}}$ & $25.6 \%$ \\
\hline & 3 of 10,000 & $-0.62(0.09)^{\mathrm{b}}$ & & $-1.39(0.07)^{\mathrm{b}}$ & & $-0.21(0.04)^{\mathrm{b}}$ & \\
\hline \multirow[t]{2}{*}{ Blood sampling twice } & No & $-0.28(0.08)^{\mathrm{b}}$ & $9.7 \%$ & $0.005(0.05)$ & $0.2 \%$ & $-0.25(0.03)^{\mathrm{b}}$ & $30.5 \%$ \\
\hline & Yes & $0.28(0.08)^{\mathrm{b}}$ & & $-0.005(0.05)$ & & $0.25(0.03)^{\mathrm{b}}$ & \\
\hline \multirow[t]{2}{*}{ Curing pneumonia without hospitalization } & 95 of 100 & $0.14(0.08)$ & $4.9 \%$ & $0.68(0.06)^{\mathrm{b}}$ & $24.3 \%$ & $0.04(0.04)$ & $5.1 \%$ \\
\hline & 90 of 100 & $-0.14(0.08)$ & & $-0.68(0.06)^{\mathrm{b}}$ & & $-0.04(0.04)$ & \\
\hline \multirow[t]{2}{*}{ Moderate muscle pain } & 5 of 100 & $0.13(0.07)$ & $4.6 \%$ & $0.52(0.05)^{\mathrm{b}}$ & $18.7 \%$ & $0.18(0.04)^{\mathrm{b}}$ & $22.1 \%$ \\
\hline & 10 of 100 & $-0.13(0.07)$ & & $-0.52(0.05)^{\mathrm{b}}$ & & $-0.18(0.04)^{\mathrm{b}}$ & \\
\hline \multirow[t]{2}{*}{ Switch of cardiovascular drug } & No & $1.68(0.12)^{\mathrm{b}}$ & $58.9 \%$ & $0.20(0.05)^{\mathrm{b}}$ & $7.0 \%$ & $-0.13(0.04)^{\mathrm{b}}$ & $16.8 \%$ \\
\hline & Yes & $-1.68(0.12)^{\mathrm{b}}$ & & $-0.20(0.05)^{\mathrm{b}}$ & & $0.13(0.04)^{\mathrm{b}}$ & \\
\hline
\end{tabular}

$R I$ relative importance, $S E$ standard error

${ }^{a}$ Average class probability

${ }^{\mathrm{b}} p<0.05$

Table 4 Results of pharmacists' latent class analysis

\begin{tabular}{|c|c|c|c|c|c|c|c|}
\hline \multirow[t]{2}{*}{ Attribute } & \multirow[t]{2}{*}{ Level } & \multicolumn{2}{|c|}{$\begin{array}{l}\text { Class 1: 'risk focused' } \\
(31.3 \%)^{\mathrm{a}}\end{array}$} & \multicolumn{2}{|c|}{$\begin{array}{l}\text { Class 2: 'stability focused' } \\
(31.1 \%)^{\mathrm{a}}\end{array}$} & \multicolumn{2}{|c|}{$\begin{array}{l}\text { Class 3: 'practicality } \\
\text { focused' }(37.6 \%)^{\mathrm{a}}\end{array}$} \\
\hline & & Utility (SE) & RI & Utility (SE) & RI & Utility (SE) & RI \\
\hline \multirow[t]{2}{*}{ Muscle damage } & 1 of 10,000 & $0.60(0.06)^{\mathrm{b}}$ & $40.6 \%$ & $0.44(0.10)^{\mathrm{b}}$ & $11.7 \%$ & $0.72(0.08)^{\mathrm{b}}$ & $20.0 \%$ \\
\hline & 3 of 10,000 & $-0.60(0.06)^{\mathrm{b}}$ & & $-0.44(0.10)^{\mathrm{b}}$ & & $-0.72(0.08)^{\mathrm{b}}$ & \\
\hline \multirow[t]{2}{*}{ Blood sampling twice } & No & $-0.06(0.06)$ & $3.8 \%$ & $0.45(0.09)^{\mathrm{b}}$ & $11.9 \%$ & $1.39(0.10)^{\mathrm{b}}$ & $38.8 \%$ \\
\hline & Yes & $0.06(0.06)$ & & $-0.45(0.09)^{\mathrm{b}}$ & & $-1.39(0.10)^{\mathrm{b}}$ & \\
\hline \multirow[t]{2}{*}{ Curing pneumonia without hospitalization } & 95 of 100 & $0.61(0.06)^{\mathrm{b}}$ & $41.4 \%$ & $0.43(0.09)^{\mathrm{b}}$ & $11.3 \%$ & $0.32(0.07)^{\mathrm{b}}$ & $8.8 \%$ \\
\hline & 90 of 100 & $-0.61(0.06)^{\mathrm{b}}$ & & $-0.43(0.09)^{\mathrm{b}}$ & & $-0.32(0.07)^{\mathrm{b}}$ & \\
\hline \multirow[t]{2}{*}{ Moderate muscle pain } & 5 of 100 & $0.17(0.06)^{\mathrm{b}}$ & $11.6 \%$ & $0.14(0.09)$ & $3.8 \%$ & $0.26(0.07)^{\mathrm{b}}$ & $7.1 \%$ \\
\hline & 10 of 100 & $-0.17(0.06)^{\mathrm{b}}$ & & $-0.14(0.09)$ & & $-0.26(0.07)^{\mathrm{b}}$ & \\
\hline \multirow[t]{2}{*}{ Switch of cardiovascular drug } & No & $0.04(0.06)$ & $2.5 \%$ & $2.31(0.20)^{\mathrm{b}}$ & $61.2 \%$ & $0.90(0.09)^{\mathrm{b}}$ & $25.2 \%$ \\
\hline & Yes & $-0.04(0.06)$ & & $-2.31(0.20)^{\mathrm{b}}$ & & $-0.90(0.09)^{\mathrm{b}}$ & \\
\hline
\end{tabular}

$R I$ relative importance, $S E$ standard error

${ }^{a}$ Average class probability

${ }^{\mathrm{b}} p<0.05$

\subsection{Pharmacists' Additional Considerations}

Eighty-six pharmacists (48\%) reported one or more situations in which their preferences would differ from those expressed in the CBC task. Clinical, risk-related situations (e.g. muscle problems in anamnesis, very serious cardiovascular disease) were reported by 64 pharmacists, issues with respect to the practicality and feasibility of the management options (e.g. switch of medication is undesirable for mentally challenged patients; blood testing is undesirable for immobile patients) by 16 , patient preferences (unwilling to switch medication, fear of adverse events, fear of needles) by 14, and prescriber preferences by five.

\section{Discussion}

Divergent preferences with regard to DDI management were observed for both patients and pharmacists. Among both patients and pharmacists, in one class highest importance was attached to risks and benefits (avoidance of serious muscle problems, cure of pneumonia). This pattern of preferences can be characterized as 'risk focused' (31.3 and $41.0 \%)$. Similarly, among both pharmacists and patients, in a second class (31.1 and 20.3\%) the highest importance was attached to avoiding changes in the current therapy ('stability focused'). For pharmacists, the third class was 'practicality focused' (37.6\%): the highest 
importance was attached to avoidance of blood sampling and to a lesser extent, like the previous class, to avoiding changes in the current therapy. In contrast, in the patients' third class $(38.7 \%)$, blood sampling was unexpectedly preferred over no blood sampling in otherwise identical situations ('certainty focused').

Pharmacists in the 'risk focused' class were older and more often male, whereas pharmacists in the 'stability focused' class were younger and more often female. This difference has parallels with the transition of pharmacy practice in recent decades, towards a more patient-oriented perspective. However, the number of pharmacists spontaneously reporting patient preferences as a reason for other DDI management considerations was limited $(n=14$ [7.9\%]).

For patients, we found no clear association between preference class and descriptive statistics, although an association with educational level could not be excluded. No relationship with health literacy was observed. About one-quarter of patients who started the questionnaire did not finish it; this included relatively more patients with lower education. Patients with low health literacy or low numeracy may have been underrepresented in our study because of the complexity of assessing the DDI management options. The mean SNS score on numeracy and the mean overall FCCHL score on health literacy in this study were in the same range as in the validation studies [27,31].

Decision making about DDIs is complex, as it includes several aspects: (1) a new or changing condition in which treatment is assumed necessary, (2) a risk of the combination of both drugs, of which the exact magnitude is often unknown, and (3) the potential management options of the DDI, which may affect both the risk and the benefit of (1) and (2) and that may also introduce new risks. We combined the characteristics of these aspects in one conjoint task, which enabled respondents to integrate the consequences of the management options. The fact that the task description focused primarily on managing the DDI may have highlighted the risk originating from the DDI (serious muscle problems). The presentation of data may have influenced the respondents in several ways. First, serious events with low risk were included as attribute. We used descriptions with absolute risks and fixed denominators for optimal understanding (e.g. 5 of 100 and 10 of 100 rather than $5 \%$ and $10 \%$ or 1 of 20 vs. 1 of 10) [34-37]. Nevertheless, understanding (rare) risks and incorporating them in healthcare decisions is difficult, especially for people with lower health literacy and lower numeracy [25, 38, 39]. Second, risk perception is strongly influenced by contextual factors, e.g. whether or not it is presented and perceived as a dangerous problem [40-42]. Third, people are sensitive to the framing of risks in terms of loss or gain. People tend to respond more strongly to options described as losses (e.g. a new serious adverse event) rather than as gains (more effective cure of pneumonia) [34]. However, this is also likely to happen in daily clinical practice.

In this context, it is interesting that the analysis showed that all patients attached importance to the risk of serious muscle problems, despite the low risk. Better chances of curing pneumonia-a serious condition-were valued less. However, this was not the case with pharmacists, who are used to risk interpretation. When it comes to differences between pharmacists and patients, the expressed preferences for the blood sampling attribute is notable. As expected, pharmacists preferred a management option solution without blood sampling, as there is no benefit in the act of blood sampling itself as long as the levels of the other attributes are identical. In fact, for one of the pharmacist classes ('practicality focused'), prevention of blood sampling was the most important factor. In contrast, in the 'certainty focused' patient class, blood testing was preferred over no blood testing. It is conceivable that these patients expected better control with blood tests, even where all other shown attribute levels were identical. A comparable effect has been observed with price, where more expensive goods were incorrectly assumed to be of better quality [43, 44]. Although the real value of the expressed preference of blood sampling over no sampling can be questioned, the results do suggest that most patients in this study, who chronically use cardiovascular drugs, do not experience blood sampling as very burdensome.

This study is not without limitations. First, we investigated a fictitious DDI. Therefore, we cannot draw conclusions about preferences in any specific situation. Patients may choose differently when they are confronted with a DDI in daily practice, and pharmacists may choose differently for specific patients. However, it is likely that preferences will also vary in daily clinical practice.

Second, invitations were sent by email. Although internet access is high in the Netherlands (in 2016: $94 \%$ of the general population; $78 \%$ in the population aged $>65$ years [45]), the oldest and frailest patients may well have been underrepresented. Moreover, patients chronically using cardiovascular drugs are often subject to DDIs, but they need not to be representative for all patients facing DDIs.

Third, the subject of our CBC was complex. Respondents not completely understanding the task may have given irrational or random answers, and identifying irrational answers is difficult [43, 44]. Some respondents may have used simplifying heuristics, ranking attributes in importance rather than making a trade-off [46]. However, it is plausible that respondents deliberately valued some attributes extremely high or low. Irrational answers may have influenced the exact estimates but are unlikely to have influenced the overall pattern. The preferred levels for all classes were consistent with prior expectations for both risk 
and benefit attributes (preference for lower risk and higher benefit).

Fourth, physicians were not included in the investigation. Although pharmacists are the healthcare providers mostly involved in DDI management, consultation with the prescriber or prescribers is an important part of the process. The physician is an essential actor in decision making and in effectuating DDI management options such as prescription modifications and monitoring laboratory values. Therefore, further investigations into DDI decision making should include all three main stakeholders: patients, pharmacists and physicians.

The observed variability in DDI management preferences can lead to incongruence between patients' and pharmacists' assessments. Awareness of their own preference and patients' preferences can help healthcare providers in shared decision making. This can be stimulated by incorporating the divergence of preferences in DDI management recommendations [47]. Further research is needed to obtain insight into the DDI decision-making process and to investigate the value and implementation of shared decision making about DDIs in daily practice.

\section{Conclusion}

Our results show considerable variability in DDI management preferences, among both patients and pharmacists. Some attach the highest importance to clinical risks and benefits, whereas others highly value practical implications (such as the acceptance or rejection of blood testing). The awareness of existing variability enables it to be incorporated into the development and application of DDI management recommendations: a step towards shared decision making in this field.

Acknowledgments The authors thank all patients and all pharmacists who participated in this study.

Author Contributions All authors contributed to the study design and the manuscript. MH coordinated the data collection, performed the data analysis and drafted the manuscript. All authors approved the final manuscript.

\section{Compliance with Ethical Standards}

Funding MH received an unconditional grant from Sawtooth Software for using Sawtooth Software Lighthouse Studio and Sawtooth Software Hosting. This research received no other specific grant from any funding agency in the public, commercial or not-for-profit sectors.

Conflicts of interest Mette Heringa, Annemieke Floor-Schreudering, Hans Wouters, Peter A.G.M. De Smet and Marcel L. Bouvy have no conflicts of interest that are directly relevant to the content of this study.
Human Subjects The Institutional Review Board of the Division of Pharmacoepidemiology and Clinical Pharmacology of Utrecht University approved the investigation, and the work was conducted in compliance with its requirements. Only anonymous data were collected. Respondents did not sign informed consent, as an anonymous survey among volunteers did not fall within the scope of the Dutch Act on Medical Research Involving Human Subjects.

Open Access This article is distributed under the terms of the Creative Commons Attribution-NonCommercial 4.0 International License (http://creativecommons.org/licenses/by-nc/4.0/), which permits any noncommercial use, distribution, and reproduction in any medium, provided you give appropriate credit to the original author(s) and the source, provide a link to the Creative Commons license, and indicate if changes were made.

\section{References}

1. Floor-Schreudering A, Geerts AF, Aronson JK, Bouvy ML, Ferner RE, De Smet PA. Checklist for standardized reporting of drug-drug interaction management guidelines. Eur J Clin Pharmacol. 2014;70:313-8.

2. Payne TH, Hines LE, Chan RC, Hartman S, Kapusnik-Uner J, Russ AL, et al. Recommendations to improve the usability of drug-drug interaction clinical decision support alerts. J Am Med Inform Assoc. 2015;22:1243-50.

3. Schoemaker CG, van der Weijden T. Patiëntvoorkeur versus evidence based medicine: hadden de pioniers van EBM oog voor wat de patiënt wil? (Patient preferences versus evidence-based medicine: did the pioneers of evidence-based medicine take the patient's preferences into account?). Ned Tijdschr Geneeskd. 2016;160:D24.

4. Greenhalgh T, Howick J, Maskrey N, Evidence Based Medicine Renaissance Group. Evidence based medicine: a movement in crisis? BMJ. 2014;348:g3725.

5. Neumann I, Santesso N, Akl EA, Rind DM, Vandvik PO, AlonsoCoello $\mathrm{P}$, et al. A guide for health professionals to interpret and use recommendations in guidelines developed with the GRADE approach. J Clin Epidemiol. 2016;72:45-55.

6. Stiggelbout AM, Van der Weijden T, De Wit MP, Frosch D, Legare F, Montori VM, et al. Shared decision making: really putting patients at the centre of healthcare. BMJ. 2012;344:e256.

7. Guyatt GH, Oxman AD, Kunz R, Falck-Ytter Y, Vist GE, Liberati $\mathrm{A}$, et al. Going from evidence to recommendations. BMJ. 2008;336:1049-51.

8. Alonso-Coello P, Oxman AD, Moberg J, Brignardello-Petersen R, Akl EA, Davoli M, et al. GRADE Evidence to Decision (EtD) frameworks: a systematic and transparent approach to making well informed healthcare choices. 2: Clinical practice guidelines. BMJ. 2016;353:i2089.

9. Scheife RT, Hines LE, Boyce RD, Chung SP, Momper JD, Sommer CD, et al. Consensus recommendations for systematic evaluation of drug-drug interaction evidence for clinical decision support. Drug Saf. 2015;38:197-206.

10. Pollard S, Bansback N, Bryan S. Physician attitudes toward shared decision making: a systematic review. Patient Educ Couns. 2015;98:1046-57.

11. Britten N, Denford S, Harris-Golesworthy F, Jibson S, Pyart N, Stein K. Patient involvement in drug licensing: a case study. Soc Sci Med. 2015;131:289-96.

12. Postmus D, Mavris M, Hillege H, Salmonson T, Ryll B, Plate A, et al. Incorporating patient preferences into drug development 
and regulatory decision making: results from a quantitative pilot study with cancer patients, carers, and regulators. Clin Pharmacol Ther. 2016;99:548-54.

13. Hazlewood GS, Bombardier C, Tomlinson G, Thorne C, Bykerk VP, Thompson A, et al. Treatment preferences of patients with early rheumatoid arthritis: a discrete-choice experiment. Rheumatol (Oxf). 2016;55:1959-68.

14. Koster ES, Blom L, Philbert D, Rump W, Bouvy ML. The Utrecht Pharmacy Practice network for Education and Research: a network of community and hospital pharmacies in the Netherlands. Int J Clin Pharm. 2014;36:669-74.

15. WHO Collaborating Centre for Drug Statistics Methodology. ATC/DDD Index 2016. Accessed August 2016. 2015; Available at: www.whocc.no/atc_ddd_index.

16. Vass C, Gray E, Payne K. Discrete choice experiments of pharmacy services: a systematic review. Int $J$ Clin Pharm. 2016;38:620-30.

17. Vass C, Rigby D, Payne K. The role of qualitative research methods in discrete choice experiments. Med Decis Making. 2017;37:298-313.

18. Reed Johnson F, Lancsar E, Marshall D, Kilambi V, Muhlbacher A, Regier DA, et al. Constructing experimental designs for discrete-choice experiments: report of the ISPOR Conjoint Analysis Experimental Design Good Research Practices Task Force. Value Health. 2013;16:3-13.

19. Bridges JF, Hauber AB, Marshall D, Lloyd A, Prosser LA, Regier DA, et al. Conjoint analysis applications in health-a checklist: a report of the ISPOR Good Research Practices for Conjoint Analysis Task Force. Value Health. 2011;14:403-13.

20. Hauber AB, Gonzalez JM, Groothuis-Oudshoorn CG, Prior T, Marshall DA, Cunningham C, et al. Statistical methods for the analysis of discrete choice experiments: a report of the ISPOR conjoint analysis good research practices task force. Value Health. 2016;19:300-15.

21. Heringa M, Floor-Schreudering A, Tromp PC, De Smet PA, Bouvy ML. Nature and frequency of drug therapy alerts generated by clinical decision support in community pharmacy. Pharmacoepidemiol Drug Saf. 2016;25:82-9.

22. Preston CL editor. Stockley's Drug Interactions (Online). London, United Kingdom: Royal Pharmaceutical Society, The Pharmaceutical Press; 2016.

23. Borgsteede SD, editor. Commentaren Medicatiebewaking 2015-2016 (Commentaries medication surveillance). 30th ed. Houten: Health Base Foundation; 2015.

24. Sawtooth Software. The CBC System for Choice-Based Conjoint Analysis (version 8). Technical Paper Series. 2013.

25. Smith SG, Curtis LM, O'Conor R, Federman AD, Wolf MS. $\mathrm{ABCs}$ or 123s? The independent contributions of literacy and numeracy skills on health task performance among older adults. Patient Educ Couns. 2015;98:991-7.

26. Malloy-Weir LJ, Charles C, Gafni A, Entwistle VA. Empirical relationships between health literacy and treatment decision making: a scoping review of the literature. Patient Educ Couns. 2015;98:296-309.

27. Zikmund-Fisher BJ, Smith DM, Ubel PA, Fagerlin A. Validation of the Subjective Numeracy Scale: effects of low numeracy on comprehension of risk communications and utility elicitations. Med Decis Making. 2007;27:663-71.

28. Dolan JG, Cherkasky OA, Li Q, Chin N, Veazie PJ. Should health numeracy be assessed objectively or subjectively? Med Decis Making. 2016;36:868-75.
29. Galesic M, Garcia-Retamero R. Do low-numeracy people avoid shared decision making? Health Psychol. 2011;30:336-41.

30. Ishikawa $\mathrm{H}$, Takeuchi $\mathrm{T}$, Yano E. Measuring functional, communicative, and critical health literacy among diabetic patients. Diabetes Care. 2008;31:874-9.

31. van der Vaart R, Drossaert CH, Taal E, ten Klooster PM, Hilderink-Koertshuis RT, Klaase JM, et al. Validation of the Dutch functional, communicative and critical health literacy scales. Patient Educ Couns. 2012;89:82-8.

32. Fagerlin A, Zikmund-Fisher BJ, Ubel PA, Jankovic A, Derry HA, Smith DM. Measuring numeracy without a math test: development of the Subjective Numeracy Scale. Med Decis Making. 2007;27:672-80.

33. Sawtooth Software. The CBC Latent Class Technical Paper (version 3). 2004.

34. Trevena LJ, Zikmund-Fisher BJ, Edwards A, Gaissmaier W, Galesic M, Han PK, et al. Presenting quantitative information about decision outcomes: a risk communication primer for patient decision aid developers. BMC Med Inf Decis Making. 2013;13(Suppl 2):S7.

35. Garcia-Retamero R, Galesic M. Using plausible group sizes to communicate information about medical risks. Patient Educ Couns. 2011;84:245-50.

36. Peters E, Hart PS, Fraenkel L. Informing patients: the influence of numeracy, framing, and format of side effect information on risk perceptions. Med Decis Making. 2011;31:432-6.

37. Griffith JM, Lewis CL, Hawley S, Sheridan SL, Pignone MP. Randomized trial of presenting absolute $\mathrm{v}$. relative risk reduction in the elicitation of patient values for heart disease prevention with conjoint analysis. Med Decis Making. 2009;29:167-174.

38. Ahmed H, Naik G, Willoughby H, Edwards AG. Communicating risk. BMJ. 2012;344:e3996.

39. Schapira MM, Fletcher KE, Gilligan MA, King TK, Laud PW, Matthews BA, et al. A framework for health numeracy: how patients use quantitative skills in health care. J Health Commun. 2008;13:501-17.

40. Ubel PA. Beyond costs and benefits: understanding how patients make health care decisions. Oncologist. 2010;15(Suppl 1):5-10.

41. Power TE, Swartzman LC, Robinson JW. Cognitive-emotional decision making (CEDM): a framework of patient medical decision making. Patient Educ Couns. 2011;83:163-9.

42. McDermott R. Medical decision making: lessons from psychology. Urol Oncol. 2008;26:665-8.

43. Lancsar E, Louviere J. Deleting 'irrational' responses from discrete choice experiments: a case of investigating or imposing preferences? Health Econ. 2006;15:797-811.

44. Ryan M, Watson V, Entwistle V. Rationalising the 'irrational': a think aloud study of discrete choice experiment responses. Health Econ. 2009;18:321-36.

45. Statistics Netherlands (Centraal Bureau voor de Statistiek, CBS). Internet: toegang, gebruik en faciliteiten (Internet; access, use and facilities), Accessed 27 October 2016. 2016; Available at: http:// statline.cbs.nl/Statweb.

46. Pieterse AH, de Vries M, Kunneman M, Stiggelbout AM, Feldman-Stewart D. Theory-informed design of values clarification methods: a cognitive psychological perspective on patient healthrelated decision making. Soc Sci Med. 2013;77:156-63.

47. van der Weijden T, Pieterse AH, Koelewijn-van Loon MS, Knaapen L, Legare F, Boivin A, et al. How can clinical practice guidelines be adapted to facilitate shared decision making? A qualitative key-informant study. BMJ Qual Saf. 2013;22:855-63. 